\title{
Exacerbation of systemic inflammation and increased cerebral infarct volume with cardiopulmonary bypass after focal cerebral ischemia in the rat
}

\author{
H. Mayumi Homi, MD, Wilbert L. Jones, MD, Fellery de Lange, MD, G. Burkhard Mackensen, MD, and \\ Hilary P. Grocott, MD, FRCPC
}

\begin{abstract}
Objective: Stroke remains a significant contributor to morbidity and mortality after cardiac surgery. Cardiopulmonary bypass is known to induce a significant inflammatory response, which could adversely influence outcomes. We hypothesized that cardiopulmonary bypass, through an enhanced systemic inflammatory response, might affect outcomes after focal cerebral ischemia.
\end{abstract}

\begin{abstract}
Methods: Wistar rats (275-300 g) were anesthetized, surgically prepared for cardiopulmonary bypass and right middle cerebral artery occlusion, and randomly allocated to 2 groups: focal cerebral ischemia alone $(\mathrm{n}=9)$ and focal cerebral ischemia combined with normothermic cardiopulmonary bypass $(n=8)$. Serum cytokines (tumor necrosis factor $\alpha$ and interleukins $1 \beta, 6$, and 10) were measured at baseline, at end of bypass, and at 2, 6, and 24 hours after bypass. On postoperative day 3 , animals underwent neurologic testing, after which the brains were prepared for assessment of cerebral infarct volume. Data were compared between groups by Mann-Whitney $U$ test.
\end{abstract}

Results: Compared with the ischemia-alone group, the ischemia plus bypass group had significantly higher levels of circulating tumor necrosis factor $\alpha$ and interleukins $1 \beta$ and 10 at the end of bypass and 2 hours after bypass. In addition, the ischemia plus bypass animals had larger total cerebral infarct volumes $\left(286 \pm 125 \mathrm{~mm}^{3}\right)$ than did those with ischemia alone $\left(144 \pm 85 \mathrm{~mm}^{3}, P=.0124\right)$.

Conclusions: Cardiopulmonary bypass increased cerebral infarct size after focal cerebral ischemia in rats. This worsening of outcome may in part be related to an augmented inflammatory response that accompanies cardiopulmonary bypass. (J Thorac Cardiovasc Surg 2010;140:660-6)

\section{Supplemental material is available online.}

Cardiac surgery is known to be associated with variable degrees of neurologic injury, ranging from relatively minor neurocognitive deficits to more severe cerebral infarction. ${ }^{1,2}$ Although the mechanisms of perioperative stroke are not fully understood, they most likely involve obstruction (by thrombus or embolus) of a cerebral vessel or prolonged cerebral hypoperfusion (particularly in a watershed area of the brain). ${ }^{3}$ As a consequence, stroke is represented by a core of irreversible neuronal necrosis surrounded by

From the Division of Cardiothoracic Anesthesiology and Critical Care Medicine, Department of Anesthesiology, Duke University Medical Center, Durham, NC.

Supported by a Society of Cardiovascular Anesthesiologists (SCA) Starter Grant (to H.M.H.).

Disclosures: None.

Received for publication June 24, 2009; revisions received Sept 8, 2009; accepted for publication Oct 8, 2009; available ahead of print March 17, 2010.

Address for reprints: Hilary P. Grocott, MD, FRCPC, Professor of Anesthesia and Surgery, University of Manitoba, I. H. Asper Institute for Clinical Research, CR3008, 369 Tache Ave., Winnipeg, Manitoba, Canada R2H 2A6 (E-mail: hgrocott@sbgh. mb.ca).

$0022-5223 / \$ 36.00$

Copyright (C) 2010 by The American Association for Thoracic Surgery doi:10.1016/j.jtcvs.2009.10.063 a near-ischemic region (penumbral area) that is potentially salvageable. ${ }^{4}$ In this penumbral region, inflammatory cells infiltrate during the earliest hours after reperfusion. Experimental and clinical data have shown that an influx of these cells leads to a local inflammatory response that can exacerbate brain injury. 5

The systemic inflammatory response that occurs during cardiac surgery, and in particular cardiopulmonary bypass (CPB), includes complement activation, endotoxemia, and the release of cytokines. ${ }^{6}$ Accordingly, many studies have demonstrated that CPB induces cytokine production, although the mechanisms for this production are complex. ${ }^{7}$ Surgical trauma, blood contact with nonendothelialized surfaces of the CPB apparatus, and reperfusion injury (to the gut, lungs, heart, and even the brain) are considered potential causes of cytokine production. ${ }^{8}$ Systemic inflammation may have effects on several organ systems. In experimental settings, inflammation is well known to contribute to cerebral ischemic injury. Clinical stroke studies have also shown a relationship between elevated cytokines and adverse outcomes, suggesting a pivotal role of inflammation in stroke outcome. ${ }^{9}$

Because CPB has a demonstrable inflammatory response and there is a known risk of stroke during cardiac surgery, we proposed to determine whether CPB influences outcome 

Abbreviations and Acronyms
$\mathrm{IL} \quad=$ interleukin
$\mathrm{CPB}=$ cardiopulmonary bypass
$\mathrm{MCAO}=$ middle cerebral artery occlusion
$\mathrm{TNF}=$ tumor necrosis factor

after focal cerebral ischemia. Specifically, we wished to discover whether the systemic inflammatory response triggered by CPB adversely affects cerebral infarct volume and functional outcome.

\section{MATERIALS AND METHODS}

The Duke University Institutional animal care and use committee approved this study, and all procedures met the National Institute of Health guidelines for animal care ("Guide for the Care and Use of Laboratory Animals," www.nap.edu/catalog/5140.html).

\section{Surgical Procedures}

Male Wistar rats (12-14 weeks old, body weight 275-300 g; Harlan Laboratories, Inc, Indianapolis, Ind) were kept fasting for 12 hours overnight but allowed free access to water before experiments. The animals were anesthetized in a chamber with $3 \%$ isoflurane in $50 \%$ oxygen, with the balance nitrogen. The trachea was intubated (14-gauge catheter), the lungs were mechanically ventilated (Harvard Rodent Ventilator; Harvard Apparatus, Boston, Mass), and the isoflurane concentration was reduced $(1.5 \%-2.0 \%)$. Ventilatory parameters were adjusted to produce an arterial $\mathrm{PCO}_{2}$ of 36 to $42 \mathrm{~mm} \mathrm{Hg}$. Pericranial temperature (measured with a needle thermistor inserted in the left temporal muscle adjacent to the skull) was servo-controlled (YSI 400 series thermistor and 73 ATA Indicating Controller; YSI Incorporated Life Sciences, Yellow Springs, Ohio) with both forced-air and surface heating systems to a defined target temperature of $37.5^{\circ} \mathrm{C}$. Rectal temperature (core temperature) was also monitored.

The animals were then prepared for $\mathrm{CPB}$ with the right superficial caudal epigastric artery cannulated (PE-10 Intramedic polyethylene tubing; BD Diagnostics, Sparks, Md) for continuous monitoring of mean arterial pressure and for arterial blood gas analysis (IL-1306 blood gas analyzer; Instrumentation Laboratory, Bedford, Mass). The ventral tail artery was cannulated, and 100 units of heparin administered (20-gauge 1.16 inch intravenous catheter Insyte-WTM; BD Medical, Medical Surgical Systems, Infusion Therapy, Sandy, Utah). This catheter was used for the arterial inflow to the CPB circuit. With a PE-50 catheter (Intramedic polyethylene tubing), the tail vein was cannulated for drug infusion during $\mathrm{CPB}$. A midline anterior cervical incision was then performed, and the right external jugular vein was identified and isolated for later cannulation. The right carotid artery was then surgically prepared for middle cerebral artery occlusion (MCAO). With blunt dissection, the right external and internal carotid arteries were identified and isolated with 5-0 silk sutures. The external carotid artery was ligated remote from its origin, and the proximal end was temporarily occluded with a microsurgical aneurysm clip, allowing subsequent intraluminal filament insertion (4-0 nylon monofilament, tip heat-blunted and coated with silicone). The jugular vein was incised, and a multiorifice dualstage venous cannula (modified from a 4.5F Desilets-Hoffman Pediatric Introducer; Cook Incorporated, Bloomington, Ind) was inserted and advanced into the right side of the heart to serve as the venous return cannula. All animals received 100 IU heparin intravenously after placement of the first cannula.

The CPB circuit was a custom-made apparatus consisting of a reservoir that collected the venous blood from the external jugular vein and was located $12 \mathrm{~cm}$ below the heart to create a gravity drainage gradient. The venous blood from the reservoir flowed to a peristaltic pump (Masterflex; Cole-Parmer Instrument Company, Vernon Hills, Ill) through silicone tubing (1.6 mm internal diameter, Tycon; Cole-Parmer) and was pumped through a small custom-made membrane oxygenator. The oxygenator consisted of 2 polymethylmethacrylate (Plexiglas; Altuglas International, Arkema Inc, Philadelphia, Pa) shells that allowed the insertion of 3 layers of diffusion membrane $\left(558 \mathrm{~cm}^{2}\right.$, total gas-exchanging surface area) glued together with epoxy in a crosswise fashion. This device was manufactured to permit blood gas exchange but also served as a heat exchanger by being attached to a system in which a continuous flow of warm water was used to maintain target blood temperature. The CPB flow was continuously monitored with a flow probe located within the circuit (2N806 flow probe and T208 volume flowmeter; Transonic Systems Inc, Ithaca, NY). The CPB circuit was primed with $10 \mathrm{~mL}$ whole blood obtained from a single heparinized (100 IU heparin intravenously per animal) and anesthetized donor rat through a cannula inserted in a right external jugular vein.

\section{Experimental Design}

After surgical preparation, the animals were allowed to stabilize for 15 minutes and then were randomly allocated to 1 of 2 groups: MCAO-alone group $(\mathrm{n}=9)$ or MCAO plus CPB group $(\mathrm{n}=8)$. MCAO was performed by insertion of the 4-0 monofilament through the external carotid artery stump. The filament was advanced until resistance was felt as the tip occluded the circle of Willis at the origin of the middle cerebral artery. The animals in the MCAO-alone group had the focal ischemia maintained for 90 minutes, after which the brain was reperfused as a consequence of filament withdrawal. In the MCAO plus CPB group, the ventilation was stopped after MCAO was established and normothermic $\left(37.5^{\circ} \mathrm{C}\right) \mathrm{CPB}$ was initiated. Isoflurane anesthesia (1.2\% inspired) was maintained during $\mathrm{CPB}$ in all groups, and atracurium $(0.3 \mathrm{mg} / \mathrm{kg}$ intravenously, repeated every 25 minutes) was administered. CPB (flow rate $160-190 \mathrm{~mL} \cdot \mathrm{kg}^{-1} \cdot \mathrm{min}^{-1}$ ) was carried out for 75 minutes. The MCAO filament was removed as in the MCAO-alone animals after 90 minutes of focal cerebral ischemia. The animals remained anesthetized and ventilated for an additional 120 minutes after $\mathrm{CPB}$ ended (or equivalent time for MCAO-alone animals). At the end of the experiment, the venous return cannula was changed to a PE-50 catheter that was implanted to allow the collection of serial blood samples after animal recovery ( 6 and 24 hours after $\mathrm{CPB}$ ). All other catheters were removed 2 hours after $\mathrm{CPB}$. The wounds were infiltrated with $1 \%$ lidocaine and closed with silk sutures. The heparin was allowed to dissipate spontaneously without protamine administration. The rats were allowed to recover in a chamber (inspired oxygen fraction 0.5 ) with a controlled environmental temperature $\left(25^{\circ} \mathrm{C}\right)$ for 24 hours before being transferred to their regular cages.

\section{Neurologic Evaluation}

Seventy-two hours after surgery, the rats underwent functional neurologic evaluation by an observer blinded to group assignment. ${ }^{10}$ This functional testing (neuroscore, in which 18 points represents normal behavior) assessed spontaneous activity as follows: no movement ( 0 points) to norma behavior ( 3 points); motor symmetry in all limbs as no movement at the left side ( 0 points) to normal behavior ( 3 points), motor symmetry in the forelimbs as no movement ( 0 points) to normal motor symmetry ( 3 points), climbing as falling trying to climb (1 point) to normal climbing (3 points), body proprioception as no response at left side (1 point) to symmetric response ( 3 points), and response to vibrissae touch as no response on the left side ( 1 point) to symmetric response ( 3 points).

\section{Serum Cytokine Analyses}

Venous blood samples were collected from the right external jugular vein just before MCAO (baseline), at the end of CPB (or equivalent time for MCAO animals), and then at 2, 6, and 24 hours after $\mathrm{CPB}$ (or equivalent 
TABLE 1. Physiologic values

\begin{tabular}{|c|c|c|c|}
\hline Parameter & $\begin{array}{r}\text { MCAO } \\
(n=9)\end{array}$ & $\begin{array}{l}\text { MCAO plus } \\
\text { CPB }(n=8)\end{array}$ & $\begin{array}{c}P \\
\text { value }\end{array}$ \\
\hline Body weight (g) & $283 \pm 10$ & $295 \pm 10$ & .0231 \\
\hline \multicolumn{4}{|c|}{ Mean arterial pressure $(\mathrm{mm} \mathrm{Hg})$} \\
\hline Baseline & $77 \pm 4$ & $75 \pm 4$ & .1625 \\
\hline $30 \mathrm{~min}$ & $74 \pm 6$ & $75 \pm 6$ & .9180 \\
\hline $90 \mathrm{~min}$ & $74 \pm 4$ & $73 \pm 4$ & .7675 \\
\hline $210 \min$ & $72 \pm 7$ & $72 \pm 8$ & .9970 \\
\hline \multicolumn{4}{|c|}{ Pericranial temperature $\left({ }^{\circ} \mathrm{C}\right)$} \\
\hline Baseline & $37.4 \pm 0.1$ & $37.4 \pm 0.1$ & .9347 \\
\hline $30 \min$ & $37.2 \pm 0.1$ & $37.1 \pm 0.2$ & .0841 \\
\hline $90 \mathrm{~min}$ & $37.4 \pm 0.1$ & $37.4 \pm 0.1$ & .3124 \\
\hline $210 \mathrm{~min}$ & $37.4 \pm 0.1$ & $37.4 \pm 0.1$ & .0962 \\
\hline \multicolumn{4}{|l|}{ Hemoglobin (g/dL) } \\
\hline Baseline & $16 \pm 1$ & $17 \pm 1$ & .4615 \\
\hline $30 \mathrm{~min}$ & $15 \pm 1$ & $14 \pm 2$ & .0461 \\
\hline $90 \mathrm{~min}$ & $15 \pm 1$ & $13 \pm 1$ & .0021 \\
\hline $210 \min$ & $15 \pm 1$ & $13 \pm 2$ & .0031 \\
\hline \multicolumn{4}{|l|}{ Hematocrit $(\%)$} \\
\hline Baseline & $39 \% \pm 2 \%$ & $39 \% \pm 2 \%$ & .7366 \\
\hline $30 \mathrm{~min}$ & $36 \% \pm 2 \%$ & $33 \% \pm 2 \%$ & .0215 \\
\hline $90 \min$ & $37 \% \pm 2 \%$ & $31 \% \pm 3 \%$ & .0004 \\
\hline $210 \mathrm{~min}$ & $36 \% \pm 1 \%$ & $31 \% \pm 3 \%$ & .0009 \\
\hline \multicolumn{4}{|l|}{ Glucose (mg/dL) } \\
\hline Baseline & $88 \pm 11$ & $75 \pm 7$ & .0077 \\
\hline $90 \mathrm{~min}$ & $98 \pm 10$ & $95 \pm 10$ & .5845 \\
\hline $210 \mathrm{~min}$ & $105 \pm 20$ & $116 \pm 13$ & .1932 \\
\hline \multicolumn{4}{|l|}{$\begin{array}{l}\text { CPB flow } \\
\quad(\mathrm{mL} /[\mathrm{kg} \cdot \mathrm{min}])\end{array}$} \\
\hline $30 \mathrm{~min}$ & - & $179 \pm 11$ & \\
\hline $90 \mathrm{~min}$ & - & $178 \pm 8$ & \\
\hline
\end{tabular}

Values are mean \pm SD. The 30- and 90-minute time points were during cardiopulmonary bypass, and the 210-minute time point was 120 minutes after cardiopulmonary bypass (middle cerebral artery occlusion-alone group had measurements at corresponding time points). $M C A O$, Middle cerebral artery occlusion, $C P B$, cardiopulmonary bypass.

time for MCAO animals). Samples were immediately centrifuged $\left(4^{\circ} \mathrm{C}\right)$, and the serum was frozen and stored $\left(-80^{\circ} \mathrm{C}\right)$ for later analysis. Serum tumor necrosis factor (TNF) $\alpha$ and interleukins (ILs) $1 \beta, 6$, and 10 were analyzed by multiplexed sandwich enzyme-linked immunosorbent assay microtiter plate according to manufacturer instructions (Endogen Search Light; Endogen, Inc, Woburn, Mass). Results were expressed as picograms per millimeter, and sensitivities for detection were $3.1 \mathrm{pg} / \mathrm{mL}$ for $\mathrm{TNF} \alpha, 3.1 \mathrm{pg} / \mathrm{mL}$ for IL1 $\beta, 6.3 \mathrm{pg} / \mathrm{mL}$ for IL6, and $0.4 \mathrm{pg} / \mathrm{mL}$ for IL10.

\section{Cerebral Infarct Volume Assessment}

After neurologic evaluation, the animals were deeply anesthetized with $5 \%$ isoflurane and decapitated. Each brain was immediately removed, immersed in methylbutane, and frozen $\left(-35^{\circ} \mathrm{C}\right)$, after which it was sectioned and the infarct volume was measured according to a previously described method. ${ }^{11}$ Briefly, coronal sections were made ( $20 \mu \mathrm{m}$ thickness), and 4 sequential slices were collected and slide mounted. The next 33 sections were discarded, and then a new 4-slice cycle was begun. The process began with the most rostral slice in which the cerebral infarct was evident and continued throughout the extent of the infarct. The sections were stained with hematoxylin and eosin. Then the brain sections were digitized $(1289 \times 960 \mathrm{ma}-$ trix of $210-\mu \mathrm{m}^{2}$ pixel units) with an image analysis system (M2 Turnkey System; Imaging Research Inc, St Catharines, Ontario, Canada). The infarct
TABLE 2. Arterial blood gas values

\begin{tabular}{|c|c|c|c|}
\hline Parameter & $\begin{array}{r}\text { MCAO } \\
(\mathbf{n}=9) \\
\end{array}$ & $\begin{array}{l}\text { MCAO plus } \\
\text { CPB }(n=8)\end{array}$ & $\begin{array}{c}P \\
\text { value }\end{array}$ \\
\hline \multicolumn{4}{|l|}{ Arterial pH } \\
\hline Baseline & $7.39 \pm 0.02$ & $7.39 \pm 0.03$ & .8633 \\
\hline $30 \mathrm{~min}$ & $7.40 \pm 0.01$ & $7.37 \pm 0.06$ & .4273 \\
\hline $90 \mathrm{~min}$ & $7.39 \pm 0.02$ & $7.38 \pm 0.03$ & .2440 \\
\hline $210 \mathrm{~min}$ & $7.38 \pm 0.04$ & $7.39 \pm 0.03$ & .3060 \\
\hline \multicolumn{4}{|c|}{ Arterial $\mathrm{PCO}_{2}(\mathrm{~mm} \mathrm{Hg})$} \\
\hline Baseline & $40 \pm 3$ & $39 \pm 4$ & .6719 \\
\hline $30 \mathrm{~min}$ & $37 \pm 4$ & $40 \pm 6$ & .4145 \\
\hline $90 \mathrm{~min}$ & $37 \pm 3$ & $42 \pm 2$ & .0491 \\
\hline $210 \mathrm{~min}$ & $40 \pm 5$ & $39 \pm 4$ & .8594 \\
\hline \multicolumn{4}{|c|}{ Arterial $\mathrm{Po}_{2}(\mathrm{~mm} \mathrm{Hg})$} \\
\hline Baseline & $171 \pm 44$ & $176 \pm 43$ & .8126 \\
\hline $30 \mathrm{~min}$ & $184 \pm 63$ & $247 \pm 76$ & .3095 \\
\hline $90 \mathrm{~min}$ & $210 \pm 55$ & $182 \pm 78$ & .4005 \\
\hline $210 \mathrm{~min}$ & $184 \pm 24$ & $228 \pm 90$ & .1806 \\
\hline \multicolumn{4}{|c|}{ Bicarbonate (mEq/L) } \\
\hline Baseline & $25 \pm 1$ & $25 \pm 2$ & .7427 \\
\hline $30 \mathrm{~min}$ & $24 \pm 3$ & $24 \pm 1$ & .8622 \\
\hline $90 \min$ & $24 \pm 2$ & $25 \pm 2$ & .0393 \\
\hline $210 \mathrm{~min}$ & $24 \pm 2$ & $25 \pm 2$ & .3129 \\
\hline \multicolumn{4}{|c|}{ Base excess (mmol/L) } \\
\hline Baseline & $0.5 \pm 1.1$ & $0.3 \pm 1.7$ & .8237 \\
\hline $30 \mathrm{~min}$ & $-0.3 \pm 1.8$ & $-0.6 \pm 1.4$ & .7638 \\
\hline $90 \mathrm{~min}$ & $-0.4 \pm 1.2$ & $0.6 \pm 0.8$ & .0611 \\
\hline $210 \min$ & $-0.2 \pm 0.8$ & $0.7 \pm 1.4$ & .0867 \\
\hline
\end{tabular}

Values are mean \pm SD. The 30 - and 90 -minute time points were during cardiopulmonary bypass, and the 210-minute time point was 120 minutes after cardiopulmonary bypass (middle cerebral artery occlusion-alone group had measurements at corresponding time points). $M C A O$, Middle cerebral artery occlusion, $C P B$, cardiopulmonary bypass.

boundaries were separately delineated for the cortical and subcortical structures by an observer (H.M.H.) blinded to group assignment. Infarct volumes were computed as running sums of infarct area multiplied by the known interval $(\mathrm{eg}, 660 \mu \mathrm{m})$ between sections over the extent of the infarct expressed as an orthogonal projection.

\section{Statistical Analysis}

Normally distributed physiologic variables and arterial blood gas data were expressed as mean $\pm \mathrm{SD}$, and differences between groups were compared with the unpaired Student $t$ test. For serum cytokine concentrations, which were not normally distributed, the nonparametric Mann-Whitney $U$ test was applied as appropriate. In addition, cerebral infarct volumes and neuroscores were compared between groups with the Mann-Whitney $U$ test. The nonparametric data were expressed as median with interquartile range.

\section{RESULTS}

Physiologic values and arterial blood gas analyses are shown in Tables 1 and 2. Few differences between groups were present (mainly related to hemoglobin and hematocrit concentrations at the end of $\mathrm{CPB}$ ). The lower values in the MCAO plus CPB animals are attributable to minor hemodilution occurring during the procedure. Also, the animals that underwent CPB had slightly lower baseline glucose serum levels than did the MCAO-alone animals (Table 1). 
TNF $\alpha$

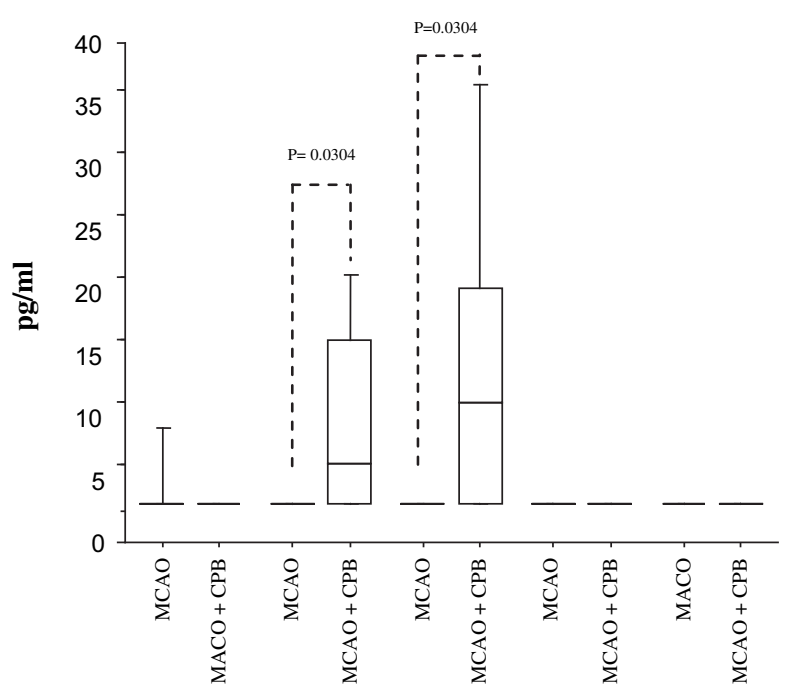

A baseline end of CPB 2hrs post $\quad 6$ hrs post $24 \mathrm{hrs}$ post

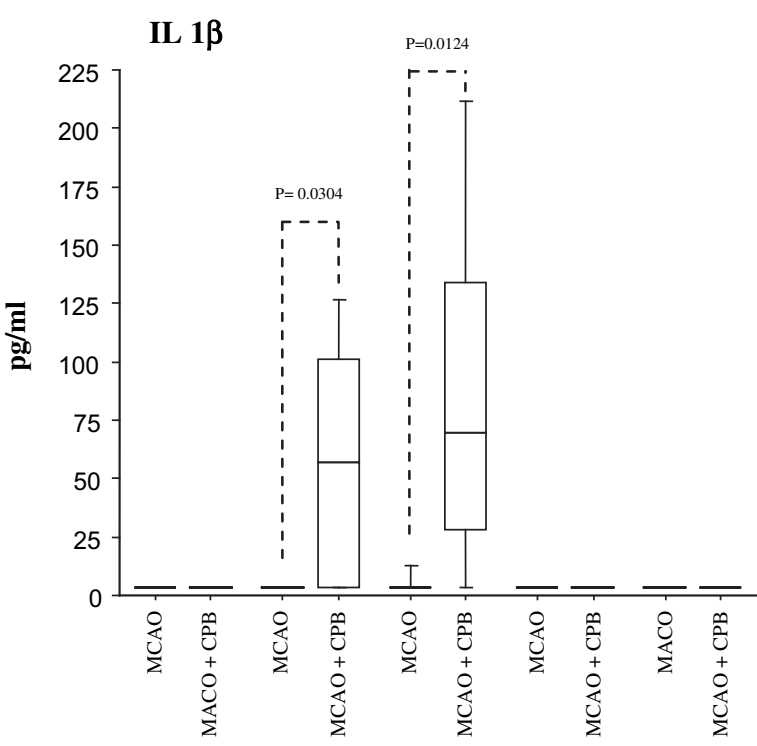

B

baseline end of CPB 2hrs post $\quad 6 h r s$ post $24 \mathrm{hrs}$ post
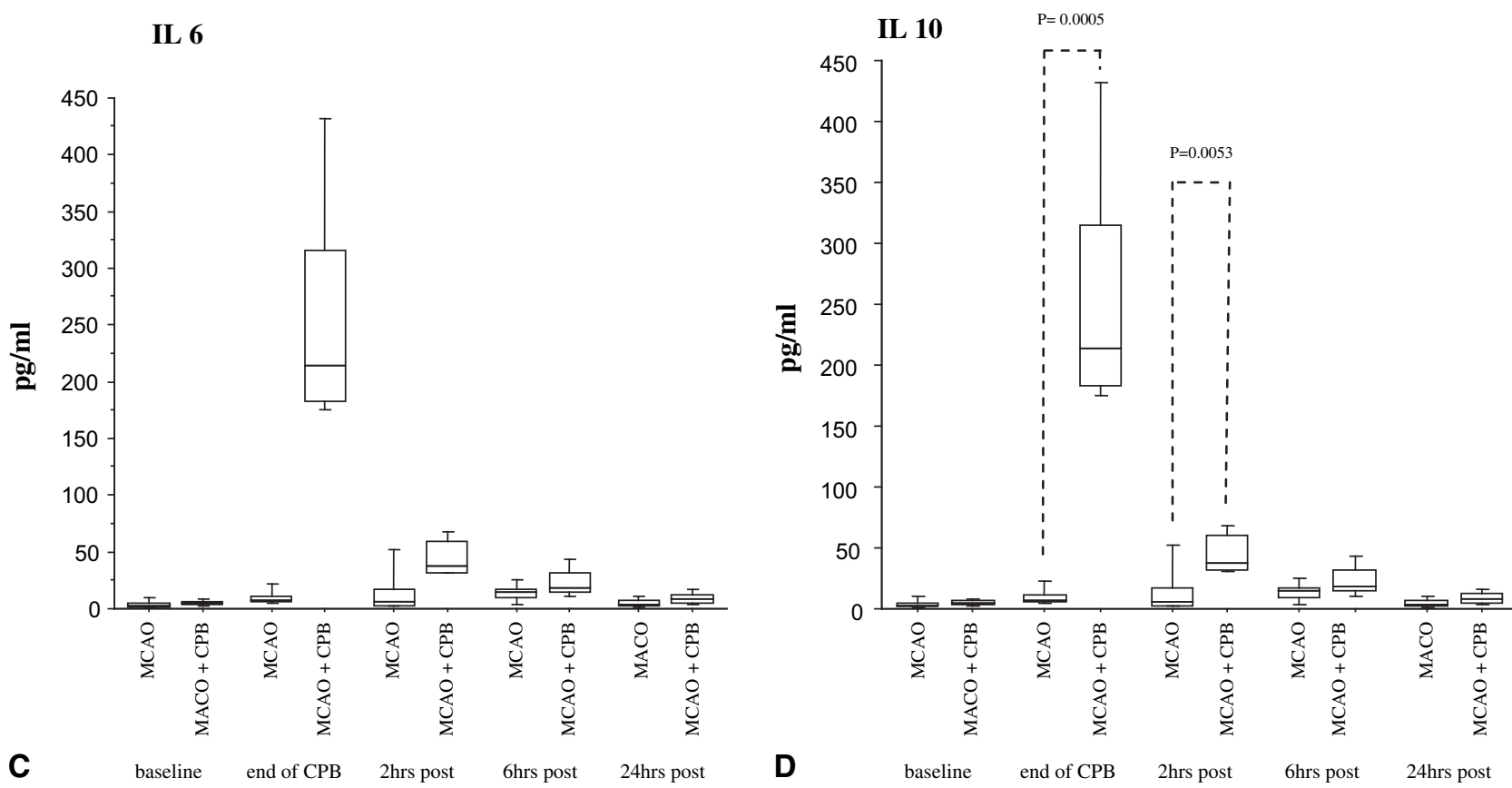

FIGURE 1. Middle cerebral artery occlusion plus cardiopulmonary bypass $(M C A O+C P B)$ group had significantly higher circulating levels of tumor necrosis factor $\alpha(\mathrm{A}, T N F \alpha)$, interleukin $1 \beta(\mathrm{B}, I L 1 \beta)$, and interleukin $10(D, I L 10)$ at end of cardiopulmonary bypass $(C P B)$ and at 2 hours after cardiopulmonary bypass relative to middle cerebral artery occlusion-alone $(M C A O)$ group. There were no differences in cytokine levels at other time points. For interleukin $6(\mathrm{C}, I L 6)$, there were no differences at any time point. Values are median with interquartile range. Asterisk indicates $P<.05$ (between-group comparisons).

The serum cytokine values were significantly different between groups. Relative to the MCAO-alone group, the MCAO plus CPB group had increased cytokine levels throughout the experiment. TNF $\alpha$ and IL1 $\beta$ had increased serum concentrations at the end of $\mathrm{CPB}$, with maximum values 2 hours after $\mathrm{CPB}$ that rapidly decreased to normal at 6 hours after CPB (Figure 1, $A$ and $B$ ). IL10 showed a sim- ilar pattern but reached its maximum concentration at the end of CPB and then decayed slowly to below a detectable threshold at 24 hours after CPB (Figure 1,D). Although the IL6 level was increased 2 hours after CPB in the MCAO plus CPB group because of high levels in just a few animals, there was no difference in median value relative to the MCAO-alone group (Figure 1, C). 


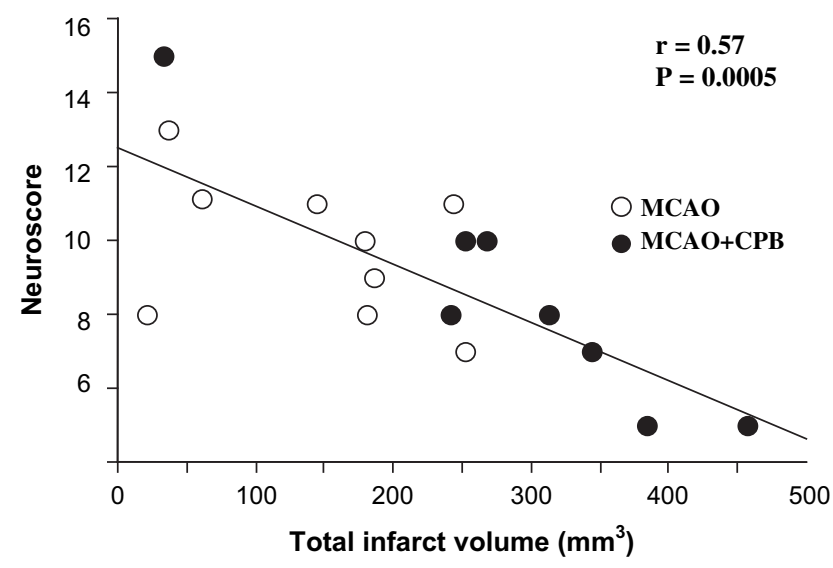

FIGURE 2. For neurologic evaluation, standard neurologic testing (neuroscore with 18 points corresponding to normal behavior) demonstrated strong correlation between neuroscore and cerebral infarct size. Neuroscore values were not significantly different between groups (median, 10 [interquartile range, 7-13] for middle cerebral artery occlusion-alone [MCAO] group vs median, 8 [interquartile range, 4-12] for middle cerebral artery occlusion plus cardiopulmonary bypass $[M C A O+C P B]$ group); however, there was trend toward worsened functional neurologic outcome in middle cerebral artery occlusion plus cardiopulmonary bypass group $(P=.1939)$.

Although there was a good correlation (Figure 2) between cerebral infarct size and worsening results of neurologic functional testing, the neuroscore values were not statistically different between groups (10 [interquartile range, 713] in MCAO group vs 8 [interquartile range, 4-12] in MCAO plus CPB group, $P=.1939$ ). In addition, there was a trend toward relationships between cytokine responses and cerebral infarct volume, but these relationships were not statistically significant. For example, the correlation between TNF $\alpha$ at the end of CPB and total cerebral infarct volume trended toward significance $\left(R^{2}=0.21, P=.07\right.$; Figure E1).

Histologic analyses of brain infarct volumes (total and cortical and subcortical components) are shown in Figure 3. The MCAO plus CPB animals had larger total cerebral infarct volumes than did MCAO-alone animals (Figure 3, $A$ ), primarily attributable to differences in cortical infarct volume (Figure 3, B).

\section{DISCUSSION}

In this study of CPB superimposed on cerebral ischemia, animals that were exposed to $\mathrm{CPB}$ had larger cerebral infarct volumes than did animals that were not exposed to CPB. This may in part have been the result of the enhanced CPB-induced inflammatory response that was demonstrated in these animals.

Wide-ranging central nervous system injuries have been reported after cardiac surgery. Focal or global brain ischemia associated with CPB is frequently implicated in these central nervous system injuries. ${ }^{2}$ Focal neurologic deficits follow
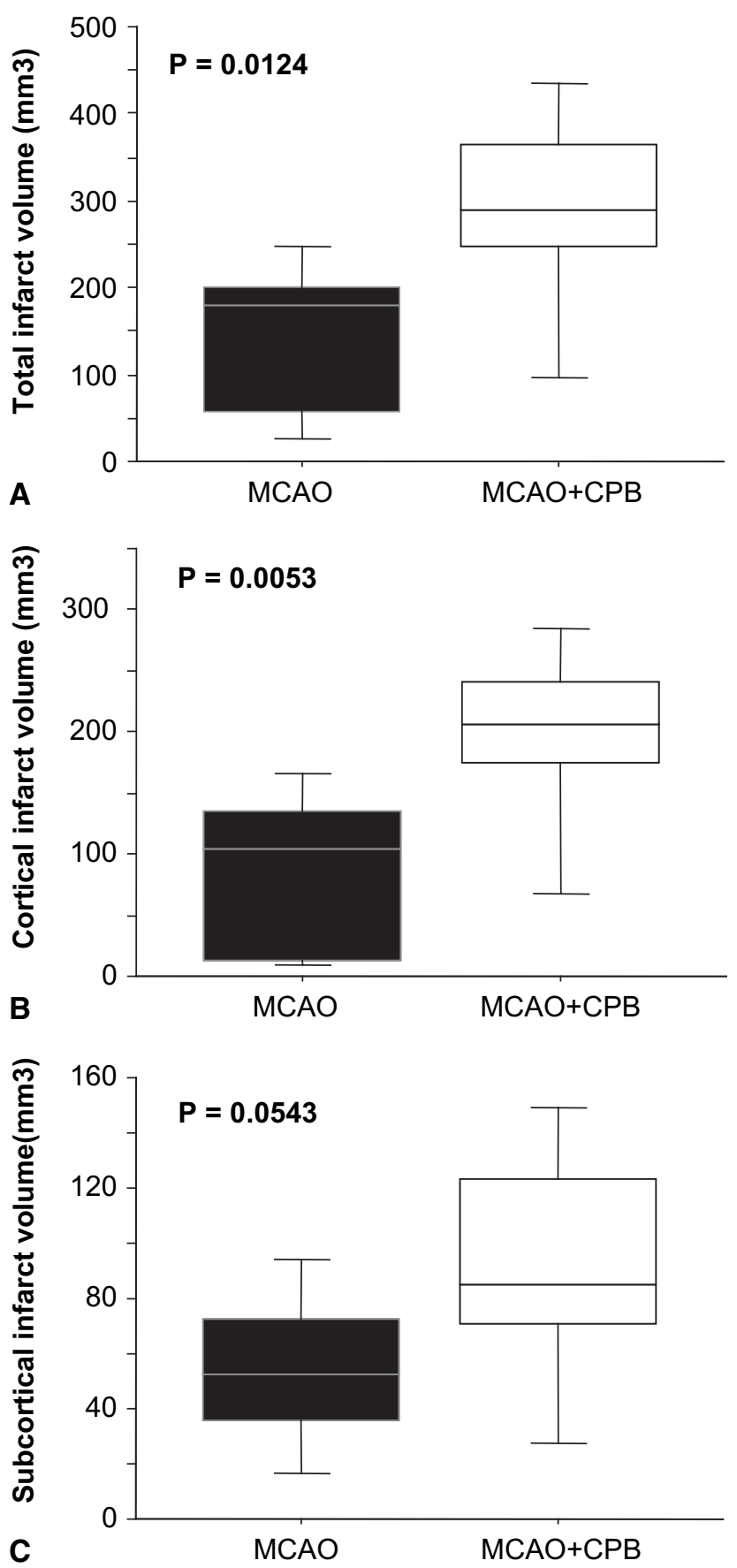

FIGURE 3. In histologic analysis, middle cerebral artery occlusion plus cardiopulmonary bypass $(M C A O+C P B)$ group had larger cerebral infarct volumes than did middle cerebral artery occlusion-alone (MCAO) group. A, Total infarct volume (middle cerebral artery occlusion-alone group $144 \pm 85 \mathrm{~mm}^{3}$ vs middle cerebral artery occlusion plus cardiopulmonary bypass group $286 \pm 125 \mathrm{~mm}^{3}$ ). B, Cortical infarct volume (middle cerebral artery occlusion-alone group $89 \pm 63 \mathrm{~mm}^{3}$ vs middle cerebral artery occlusion plus cardiopulmonary bypass group $195 \pm 81 \mathrm{~mm}^{3}$ ). C, Subcortical infarct volume (middle cerebral artery occlusion-alone group $55 \pm 28 \mathrm{~mm}^{3}$ vs middle cerebral artery occlusion plus cardiopulmonary bypass group $90 \pm$ $45 \mathrm{~mm}^{3}$ ). Values are mean \pm SD. 
cerebral arterial occlusion by macroemboli (blood clots and atheromatous or other large particulate debris) or thrombosis in cerebral arterial vessels. ${ }^{3}$ Moreover, microemboli (variably composed of air, fat, thrombus, and atheromatous fragments) in distal cerebral arteries have been related to stroke as well as to neurocognitive dysfunction. ${ }^{12}$ In addition to embolization, inflammation, either cerebral or systemic, has also been implicated in CPB-related cerebral injury. ${ }^{2}$

The inflammatory response occurring after cardiac surgery is initiated by several factors, including blood contact with artificial surfaces in the CPB circuit, surgical trauma, ischemia-reperfusion injury, and endotoxemia. Although measurement of cytokine levels has been widely used to assess inflammation after $\mathrm{CPB}$, the relationship between the magnitude of these markers and organ dysfunction has not been well established. Cytokines modulate local inflammatory responses but also have systemic effects. ${ }^{13}$ The sources of cytokine production after CPB are the heart, lungs, gut, and brain. ${ }^{14}$ In each of these organs, increased local concentrations of cytokines have been associated with heart failure, prolonged mechanical ventilatory support, endotoxemia, and brain injury. ${ }^{9,15}$ Products of increased local cytokine production may migrate into the systemic circulation and could further trigger a systemic inflammatory response.

In addition to the risk of focal cerebral ischemia, patients undergoing cardiac surgery also face the superimposition of variable degrees of inflammation in the perioperative period. In a recent clinical study, increases in cytokines and soluble TNF receptor 1 were associated with brain injury in patients who underwent CPB. ${ }^{9}$ In that study, several serum inflammatory markers were measured in patients who had postoperative cerebral damage and compared with those in patients without stroke. Serum levels of TNF $\alpha$ and IL10 were higher in the stroke group at 12 and 24 hours after CPB. Although IL 6 levels in the stroke group were higher than those in the group without stroke, these values were not statistically different. The study's authors suggested the possibility that the increased cytokines levels in patients with stroke may be associated with augmentation of inflammatory responses in the injured brain during or after $\mathrm{CPB} .{ }^{9}$

The mechanism by which enhanced inflammation may have an impact is likely related to the susceptibility of the penumbral area to damage by inflammatory cells. ${ }^{16}$ After focal brain ischemia, there is an influx of inflammatory cells to the penumbral area. ${ }^{17}$ These cells migrate to this region and infiltrate the brain parenchyma after mediation by adhesion molecules that are, at least in part, affected by proinflammatory cytokines. ${ }^{18}$ It is logical to speculate that CPB-induced cytokine production might amplify this initial response.

In our study, proinflammatory cytokines TNF $\alpha$ and IL $1 \beta$ were increased during CPB. They continued to increase after cessation of CPB and then began to decrease toward baseline values. The IL10 levels, representing a counteractive anti-inflammatory process, were in accordance with previous results that showed a phased increase and then a slow decrement temporally related to $\mathrm{CPB} .{ }^{13}$ Although IL6 peaked at 2 hours after CPB in the MCAO plus CPB group, we were unable to demonstrate any differences at the time points studied. IL6 is considered a potent proinflammatory cytokine but has a short half-life in plasma. ${ }^{13}$ Our scheduled sampling time points for cytokine screening may have precluded the detection of transient IL6 increases.

There were some limitations of this study. Minor physiologic differences were present between the groups related to hematocrit and glucose. Mild hemodilution occurred during $\mathrm{CPB}$, in the final stages of the $\mathrm{MCAO}$, and early during reperfusion. This may have served to mitigate some of the injury that was seen, because mild to moderate levels of hemodilution have previously been associated with neuroprotection after brain ischemia. ${ }^{19}$ Experimental and clinical studies have described an optimal hematocrit of approximately $30 \%$ as the most favorable level for protection from focal cerebral ischemia. ${ }^{20,21}$ Our study showed hematocrit values near this optimal set point after CPB. With respect to glucose, baseline values were marginally higher (only $13 \mathrm{mg} / \mathrm{dL}$ ) in the MCAO-alone group. In clinical and experimental settings, higher glucose levels have been extensively associated with larger brain infarcts and poor neurologic outcome. ${ }^{22}$ If anything, however, this increase in glucose in the MCAO-alone group would have served to decrease differences between groups.

A second limitation of this study is related to the need for the addition of blood to prime the CPB system. The CPB system was blood primed in an attempt to maintain similar hemoglobin levels between groups, thus avoiding confounding factors that could be caused by excessive hemodilution. Transfusion has been a variable risk factor for adverse outcome after $\mathrm{CPB}$; however, confounding this risk is the age of the products transfused. ${ }^{23}$ Because we used fresh blood donors, it is unlikely that this was a significant factor in our results. Other CPB experiments performed in our laboratory make it unlikely that the blood prime, rather than $\mathrm{CPB}$ itself, had an effect on the enhanced inflammation in the CPB group. Animals that have undergone bloodless priming of the circuit have demonstrated a similar inflammatory response pattern (unpublished data). Another limitation relates to the stroke model itself. MCAO was produced in these animals by filament insertion through the carotid artery. This is different from the mechanism of stroke occurring during clinical $\mathrm{CPB}$, in which it is more likely that embolic material consists of either thrombus or atherosclerotic material. As a result, the cerebral ischemia produced in our study was transient, whereas in the clinical setting it is more likely to be permanent.

Although these experiments were performed with strictly normothermic $\mathrm{CPB}$, in clinical situations, it is more likely for tepid temperatures to be used. As a result, application 
of these results to true hypothermic $\left(32^{\circ}-34^{\circ} \mathrm{C}\right) \mathrm{CPB}$ conditions may not be appropriate. That said, the trend toward using less significant degrees of hypothermia makes this limitation arguably minor.

The relatively short postoperative follow-up may represent a final potential limitation. There are 2 reasons we chose postoperative day 3 to analyze the cerebral infarct size in our protocol. First, the systemic inflammation, as measured by serum cytokines, was undetectable 24 hours after surgery. We can assume that the systemic component of the inflammation will not have any effect in the brain 3 days after surgery. Also, in other MCAO studies in rats the infarct volume, although changing significantly between 6 and 72 hours, had little change after that. ${ }^{17}$ Moreover, studies have shown that neutrophil infiltration in the ischemic hemisphere starts as early as 1 hour after reperfusion, increasing significantly by 6 to 12 hours. ${ }^{24}$ Others have demonstrated that the number of neutrophils peaks between 24 and 72 hours and then decreases sharply. ${ }^{25}$ After 3 days, the ischemic tissue follows progressive stages of matrix organization for approximately 2 weeks, when the necrotic tissue is removed and glial scar is formed. ${ }^{24}$

Normothermic CPB appears to affect neurologic outcome adversely after focal cerebral ischemia induced by reversible MCAO. We suggest that these results are at least partially attributable to the systemic inflammatory response triggered by CPB. The association between the systemic inflammatory response after $\mathrm{CPB}$ and deleterious neurologic outcome indicates that this response may provide a target for neuroprotective strategies.

\section{References}

1. Newman MF, Kirchner JL, Phillips-Bute B, Gaver V, Grocott H, Jones RH, et al. Longitudinal assessment of neurocognitive function after coronary-artery bypass surgery. N Engl J Med. 2001;344:395-402. Erratum in: N Engl J Med. 2001;344: 1876.

2. Hindman BJ, Todd MM. Improving neurologic outcome after cardiac surgery. Anesthesiology. 1999;90:1243-7.

3. Stump DA, Rogers AT, Hammon JW, Newman SP. Cerebral emboli and cognitive outcome after cardiac surgery. J Cardiothorac Vasc Anesth. 1996;10:113-9.

4. Garcia JH, Liu KF, Ho KL. Neuronal necrosis after middle cerebral artery occlusion in Wistar rats progresses at different time intervals in the caudoputamen and the cortex. Stroke. 1995;26:636-43.

5. Barone FC, Feuerstein GZ. Inflammatory mediators and stroke: new opportunities for novel therapeutics. J Cereb Blood Flow Metab. 1999;19:819-34.

6. Levy JH, Tanaka KA. Inflammatory response to cardiopulmonary bypass. Ann Thorac Surg. 2003;75:S715-20.
7. Rothenburger M, Soeparwata R, Deng MC, Schmid C, Berendes E, Tjan TD, et al. Prediction of clinical outcome after cardiac surgery: the role of cytokines, endotoxin, and anti-endotoxin core antibodies. Shock. 2001;16(Suppl. 1):44-50.

8. Paparella D, Yau TM, Young E. Cardiopulmonary bypass induced inflammation: pathophysiology and treatment. An update. Eur J Cardiothorac Surg. 2002;21: 232-44.

9. Nakamura K, Ueno T, Yamamoto H, Iguro Y, Yamada K, Sakata R. Relationship between cerebral injury and inflammatory responses in patients undergoing cardiac surgery with cardiopulmonary bypass. Cytokine. 2005;29:95-104.

10. Bederson JB, Pitts LH, Tsuji M, Nishimura MC, Davis RL, Bartkowski H. Rat middle cerebral artery occlusion evaluation of the model and development of a neurologic examination. Stroke. 1986;17:472-6.

11. Homi HM, Yokoo N, Ma D, Warner DS, Franks NP, Maze M, et al. The neuroprotective effect of xenon administration during transient middle cerebral artery occlusion in mice. Anesthesiology. 2003;99:876-81.

12. Pugsley W, Klinger L, Paschalis C, Treasure T, Harrison M, Newman S. The impact of microemboli during cardiopulmonary bypass on neuropsychological functioning. Stroke. 1994;25:1393-9.

13. McBride WT, Armstrong MA, Crockard AD, McMurray TJ, Rea JM. Cytokine balance and immunosuppressive changes at cardiac surgery: contrasting response between patients and isolated CPB circuits. Br J Anaesth. 1995;75:724-33.

14. Nandate K, Vuylsteke A, Crosbie A, Messahel S, Oduro-Dominah A, Menon D. Cerebrovascular cytokine responses during coronary artery bypass surgery: specific production of interleukin- 8 and its attenuation by hypothermic cardiopulmonary bypass. Anesth Analg. 1999;89:823-8.

15. Rothenburger M, Tjan TD, Schneider M, Berendes E, Schmid C, Wilhelm MJ, et al. The impact of the pro- and anti-inflammatory immune response on ventilation time after cardiac surgery. Cytometry B Clin Cytom. 2003;53:70-4.

16. Kochanek PM, Hallenbeck JM. Polymorphonuclear leukocytes and monocytes/ macrophages in the pathogenesis of cerebral ischemia and stroke. Stroke. 1992; 23:1367-79.

17. Stoll G, Jander S, Schroeter M. Inflammation and glial responses in ischemic brain lesions. Prog Neurobiol. 1998;56:149-71.

18. Joyce DE, Nelson DR, Grinnell BW. Leukocyte and endothelial cell interactions in sepsis: relevance of the protein C pathway. Crit Care Med. 2004;32(5 Suppl): S280-6.

19. Hypervolemic hemodilution treatment of acute stroke. Results of a randomized multicenter trial using pentastarch. The Hemodilution in Stroke Study Group. Stroke. 1989;20:317-23.

20. Cole DJ, Drummond JC, Shapiro HM, Hertzog RE, Brauer FS. The effect of hypervolemic hemodilution with and without hypertension on cerebral blood flow following middle cerebral artery occlusion in rats anesthetized with isoflurane. Anesthesiology. 1989;71:580-5.

21. Cole DJ, Drummond JC, Patel PM, Reynolds LR. Hypervolemic-hemodilution during cerebral ischemia in rats: effect of diaspirin cross-linked hemoglobin (DCLHb) on neurologic outcome and infarct volume. J Neurosurg Anesthesiol. 1997;9:44-50

22. Schurr A. Bench-to-bedside review: a possible resolution of the glucose paradox of cerebral ischemia. Crit Care. 2002;6:330-4.

23. Koch CG, Li L, Sessler DI, Figueroa P, Hoeltge GA, Mihaljevic T, et al. Duration of red-cell storage and complications after cardiac surgery. N Engl J Med. 2008; 358:1229-39.

24. Clark RK, Lee EV, White RF, Jonak ZL, Feuerstein GZ, Barone FC. Reperfusion following focal stroke hastens inflammation and resolution of ischemic injured tissue. Brain Res Bull. 1994;35:387-92.

25. Matsuo Y, Onodera H, Shiga Y, Nakamura M, Ninomiya M, Kihara T, et al. Correlation between myeloperoxidase-quantified neutrophil accumulation and ischemic brain injury in the rat. Effects of neutrophil depletion. Stroke. 1994; 25:1469-75. 


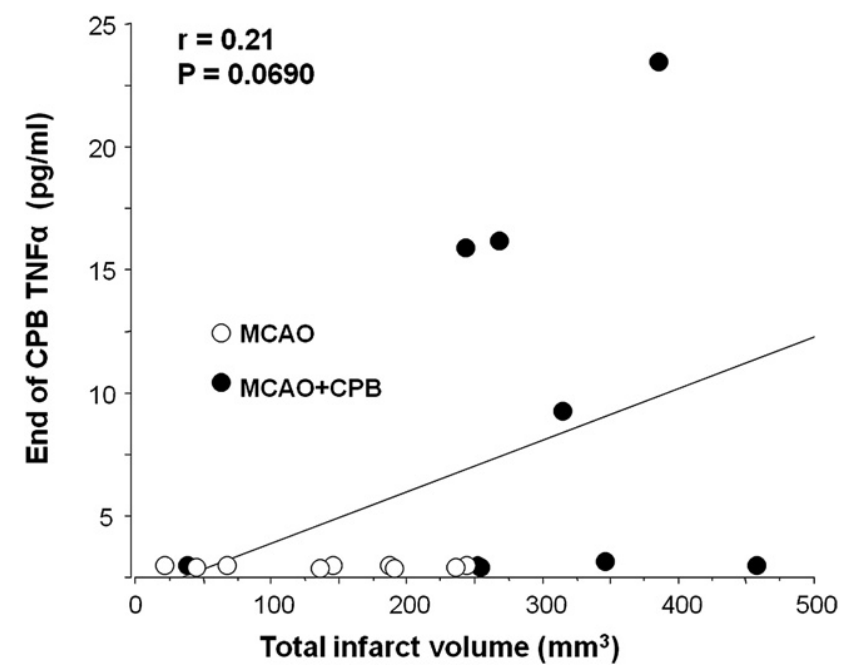

FIGURE E1. Correlation between inflammatory cytokine tumor necrosis factor $\alpha(T N F \alpha)$ levels at end of cardiopulmonary bypass $(C P B)$ with total cerebral infarct volume on postoperative day 3. $M C A O$, Middle cerebral artery occlusion-alone; $M C A O+C P B$, middle cerebral artery occlusion plus cardiopulmonary bypass. 